\title{
Recommended curriculum for subspecialty training in transplant infectious disease on behalf of the American Society of Transplantation Infectious Diseases Community of Practice Educational Initiatives Working Group
}

R. Avery, H. Clauss, L. Danziger-Isakov, J. Davis, K. Doucette, D. van Duin, J. Fishman, F. Gunseren, A. Humar, S. Husain, C. Isada, K. Julian, D. Kaul, D. Kumar, S. Martin, M. Michaels, M. Morris, F. Silveira, A. Subramanian. Recommended curriculum for subspecialty training in transplant infectious disease on behalf of the American Society of Transplantation Infectious Diseases Community of Practice Educational Initiatives Working Group.

Transpl Infect Dis 2010: 12: 190-194. All rights reserved

Abstract: The American Society of Transplantation Infectious Diseases (ID) Community of Practice has established an education workgroup to identify core components of a curriculum for training specialists in transplant ID. Clinical, laboratory, and research training form the triad of components on which an additional year of ID training, dedicated to the care of solid organ and hematopoietic stem cell transplant recipients, should be based. The recommended training environment would have access to adequate numbers of transplant patients, along with qualified faculty committed to teaching specialized fellows in this area. The learning objectives for both inpatient and outpatient clinical training are presented. The laboratory component requires trainees to attain expertize in utilizing and interpreting cutting-edge diagnostics used in transplant medicine. The research component may involve basic science, and translational or clinical research individualized to the trainee. Finally, suggestions for evaluation of both the fellows and the training program are provided.

\author{
R. Avery ${ }^{1}$, H. Clauss ${ }^{2}$, L. Danziger-Isakov', \\ J. Davis' ${ }^{3}$ K. Doucette ${ }^{4}$, D. van Duin ${ }^{1}$, \\ J. Fishman ${ }^{5}$, F. Gunseren ${ }^{6}$, A. Humar', \\ S. Husain ${ }^{7}$, C. Isada ${ }^{1}$, K. Julian ${ }^{8}$, D. Kaul ${ }^{9}$, \\ D. Kumar ${ }^{4}$, S. Martin ${ }^{3}$, M. Michaels ${ }^{10}$, \\ M. Morris ${ }^{11}$, F. Silveira ${ }^{12}$, A. Subramánian ${ }^{13}$ \\ ${ }^{1}$ Cleveland Clinic Hospital, Cleveland, Ohio, USA, ${ }^{2}$ Temple \\ University Hospital, Philadelphia, Pennsylvania, USA, ${ }^{3}$ Ohio \\ State University Hospitals, Columbus, Ohio, USA, ${ }^{4}$ University \\ of Alberta, Edmonton, Alberta, Canada, ${ }^{5}$ Massachusetts \\ General Hospital, Boston, Massachusetts, USA, ${ }^{6}$ Akdeni \\ University, Antalya, Turkey, ${ }^{7}$ Toronto General Hospital, \\ Toronto, Ontario, Canada, ${ }^{8}$ Pennsylvania State University \\ College of Medicine, Hershey, Pennsylvania, USA, ${ }^{9}$ University \\ of Michigan Hospitals, Ann Arbor, Michigan, USA, \\ ${ }^{10}$ Children's Hospital of Pittsburgh, Pittsburgh, Pennsylvania, \\ USA, ${ }^{11}$ University of Miami Miller School of Medicine, \\ Miami, Florida, USA, ${ }^{12}$ University of Pittsburgh Hospital, \\ Pittsburgh, Pennsylvania, USA, ${ }^{13}$ Johns Hopkins Hospital, \\ Baltimore, Maryland, USA
}

Key words: curriculum; training; subspecialty; transplant infectious disease

Correspondence to:

Aruna Subramanian, MD, Transplant and Oncology Center, Division of Infectious Diseases, Johns Hopkins University School of Medicine, 1830 E. Monument St, Room 463A, Baltimore, MD 21287, USA

Tel: 4106146431

Fax: 4106148488

E-mail: asubra@jhmi.edu

Received 2 March 2010, accepted for publication 4 March 2010

DOI: 10.1111/j.1399-3062.2010.00510.x

Transpl Infect Dis 2010: 12: 190-194 
The subspecialty of Transplant Infectious Disease (ID) represents a unique knowledge base that differs from 'general' ID. ID fellows who wish to pursue a career path in which a significant portion of their duties includes the care of the transplant patient should pursue additional training in transplant ID. The American Society of Transplantation (AST) ID Community of Practice has developed the following set of recommendations that outline the key components of an adult transplant ID fellowship program, which can be further adapted for pediatric-specific training. This curriculum is based on a document originally developed by Drs Robin Avery and Jay Fishman for training fellows in transplant ID, which has been modified by the AST ID Community of Practice Educational Initiatives Working Group.

\section{Training program}

\section{Duration of recommended training program}

The minimum recommended duration of additional training to attain clinical competence in the field of transplant ID is 1 year. This training is to be pursued after the trainee has completed his/her basic training in general ID. The duration of transplant ID training, however, will vary based upon the specific goals of the trainee, particularly if research is a major career goal.

\section{Training environment}

It is recognized that transplant programs vary widely in terms of types of transplant procedures performed, volume of transplantation, and clinical practices. The following environment is preferred to ensure comprehensive training of the fellow:

- An active multi-organ transplant program with sufficient volume to allow the trainee to see common opportunistic pathogens observed in these patients, and an active allogeneic hematopoietic stem cell transplant (HSCT) program is preferred.

- Fewer centers have large lung transplant programs, and training in this area may therefore be more difficult to achieve. Because lung transplant and HSCT patients are often at the greatest risk for infectious complications, periods of elective training should be considered at another center, if such training is not available at the primary training site.

- The center should have at least 1 physician (and preferably a group of physicians) with clear expertise in transplant ID to ensure adequate mentorship.
- The center should have established didactic and casebased interactive teaching conferences that encourage trainee participation and that encompass both clinical and research educational objectives.

\section{Components}

Major components

Three major components are recommended for a 1-year standard training curriculum in transplant ID.

(1) Clinical training (24 week minimum).

(2) Laboratory training (2-4 week minimum).

(3) Research training (12-20 weeks).

\section{Additional training components}

In addition to the clinical, laboratory, and research training, standard training competencies in practice-based learning, professionalism, ethics, and systems development related to transplant ID should be demonstrated. The program should assess the following objectives:

- Practice-based learning:

- Develop knowledge of fundamental resources including, but not limited to, the AST Transplant ID guidelines, evidence-based medicine databases, and additional guidelines from other critical sources. A list of key references and supplementary teaching materials, developed by the AST ID Community of Practice, is available on the internet (http://www.a-s-t.org/index.cfm).

- Utilize these resources to participate in case-based learning with transplant ID faculty.

- Professionalism/communication:

- Develop skills to interact appropriately with patients and families while respecting patient confidentiality.

- Demonstrate cooperative team building with all members of the transplant team including nurses, coordinators, data managers, and physicians/surgeons.

- Understand how the transplant ID physician interacts with the multi-disciplinary transplant team.

- The trainee should come to understand that transplant ID forms an integral component of the transplant program and transplant-related care.

- Ethics:

- Understand the processes of consent, donor, and recipient selection (including issues of brain death and donation after cardiac death), allocation systems, and considerations regarding re-transplantation and limited resource utilization. 
- Systems development:

- In the United States, understand the structure and function of OPTN/UNOS, OPOs, and the National Marrow Donor Program (in Canada, the Stem Cell and Marrow Network).

- Develop an understanding for the need and methods to increase donation at large and in minority groups.

\section{Detailed description of core components}

Clinical training. The clinical component of training should include both inpatient and outpatient care. The clinical training should be focused on transplant patients (solid organ transplant [SOT], HSCT) including pre-transplant assessment and peri-operative and post-transplantation care. Training programs may also encompass ID care for patients immunocompromised on the basis of cancer chemotherapy. Care of other immunocompromised hosts, such as patients with human immunodeficiency virus, should not be a significant focus of training unless transplant-related. Inpatient clinical service. Inpatient clinical service is the core of the transplant ID training program. The duration of inpatient service commitments will vary depending on the career goals of the fellow, but a minimum of 24 weeks is recommended. Clinical encounters should occur on inpatient wards, intensive care units, and the emergency department. Each case should be reviewed by the attending physicians, and important learning points highlighted and discussed on a case-by-case basis. Fellows should be expected to provide longitudinal follow-up of patients along with the attending physicians. Independent decision making and problem solving should be encouraged. Medical decision making should take into account relevant findings (Gram stain, preliminary culture results, molecular diagnostic assays, pathology and imaging results, etc.) from the Clinical Microbiology Laboratory, and the Pathology and Radiology Departments.

Outpatient clinic. The fellow should have an outpatient component to clinical training. This requirement is more flexible, but preferably should be 1 or 2 longitudinal half-day clinics per week. All cases should be reviewed with the attending.

\section{Learning objectives: clinical}

\section{Major goals}

(1) Understand how transplant ID fits into the global context of transplant care, from pre-transplant workup and transplant selection committees, to donor evaluation and peri-operative care, to post-transplant management.
(2) Understand immunosuppression, and how specific immunosuppressive agents contribute to the net state immunosuppression. This includes knowledge of immunosuppressive mechanisms of action, drug interactions with anti-infectives including enhanced toxicity with some combinations of antimicrobials and immunosuppressives, evolving assays to assess immune function, and infectious risk with enhanced immunosuppression related to induction therapy or treatment of rejection.

(3) Identify the major pathogens of opportunistic and other common infections as well as the major clinical syndromes that occur in SOTand HSCT recipients.

(4) Understand the principal diagnostic modalities including radiology, microbiology (including molecular and immunologic diagnostic assays), and histopathology, along with the most effective application and limitations of each in this patient population.

(5) Understand strategies for prevention of infection in these patients including the use of prophylactic and preemptive strategies.

(6) Understand the principles of treatment of infections in these patients, and how these patients differ from nonimmunocompromised hosts in terms of therapeutic response and therapeutic urgency.

(7) Become comfortable in dealing with life-threatening infections and critically ill transplant patients and their families.

Specific objectives: SOT

- Describe the approach to and treatment of common infections observed in patients with end-stage organ failure. Specifically, this includes, but is not limited to:

- infections related to dialysis access in patients with end-stage renal disease,

- spontaneous bacterial peritonitis and hepatitis $\mathrm{B}$ and $\mathrm{C}$ in patients with liver failure,

- exacerbations of cystic fibrosis in patients awaiting lung transplantation,

- infections related to mechanical circulatory support including extracorporeal membrane oxygenation and ventricular assist devices in cardiac transplant candidates.

- Perform complete pre-transplantation evaluations.The fellow should understand the risks for infection after transplant based on epidemiology and personal history, and communicate these risks to potential recipients. This workup should include an evaluation of the need for immunization using medical history and/or laboratory data. 
- Have a clear approach to common donor-related ID and current laboratory evaluation techniques for potentially transmissible infections in donors. In the United States, understand the reporting requirements of donor-transmitted infections.

- Describe technical complications and infectious sequelae that may occur as a result of such complications. The trainee should have a fluent understanding of the anatomy related to the transplant procedure. The trainee should have a clear understanding of the incidence, risk factors, timing, and presentations of vascular and non-vascular technical complications related to each transplant procedure.

- Appreciate the risks associated with hospitalization at transplant, including but not limited to infection control and prevention policies, hand hygiene, surgical site infections, and nosocomial infections related to instrumentation.

- Understand the approach to the transplant patient with fever and no localizing signs. This approach includes use of appropriate imaging and laboratory diagnostics.

- For disease-specific presentations, understand the appropriate medical history including potential exposures, physical examination, imaging, and laboratory diagnostics. Commonly encountered diseases that should be covered include viral infections (cytomegalovirus [CMV], Epstein-Barr virus [EBV], varicella zoster virus [VZV], other herpesviruses, BK virus, hepatitis viruses, and community-acquired respiratory viruses), mycobacterial infections (tuberculosis, non-tuberculous mycobacteria), fungal infections (Candida, Aspergillus, and rare molds), and bacterial infections (including emerging multi-drug resistant bacteria: Pseudomonas, carbapenem-resistant Klebsiella pneumoniae, Acinetobacter baumannii, methicillinresistant Staphylococcus aureus, and vancomycin-resistant Enterococcus).

- Perform comprehensive follow-up for infectious episodes in the outpatient setting.

- Understand outpatient evaluation for transplant recipients planning travel and the need for continued review of immunization status after transplantation.

\section{Specific objectives: HSCT/oncology}

- Demonstrate understanding of the pre-transplantation clinical assessment, including risk based on HSCT modality (myeloablative vs. reduced-intensity, allogeneic vs. autologous, related, unrelated, and cord blood donors), and donor assessment including vaccination and disqualification.
- Exhibit understanding of peri-transplant prophylaxis strategies in the induction, neutropenic, and early engraftment phases.

- Appreciate the risks associated with hospitalization at transplant, including but not limited to infection control and prevention policies, hand hygiene, surgical site infections, and infection related to instrumentation.

- Discuss the diagnosis and management of early posttransplant infectious complications including viral infections (CMV, EBV, VZV, other herpesviruses, BK virus, adenovirus, and community-acquired respiratory viruses), fungal infections (Candida, Aspergillus, and rare molds), and bacterial infections (including emerging multi-drug-resistant bacteria).

- Demonstrate understanding of late post-transplant complications including graft-versus-host disease and autoimmune diseases.

- Appreciate the difficulty in discerning graft-versushost disease from infection in this population.

- Describe the recommendations and rationale for revaccination after HSCT.

\section{Laboratory component}

Modern laboratory methods, specifically molecular diagnostics, are a key component of transplant ID practice and training. The trainee should spend time in a modern molecular diagnostic laboratory that services the transplant population. The trainee should understand the methodology, interpretations, and limitations of molecular testing for pathogens, specifically CMV, EBV, and BK virus.

\section{Objectives}

- To attain a basic understanding of laboratory techniques that are fundamental to transplant virology, including sterile technique, centrifuging, pipetting, running gels, making media, filtering, maintenance of cell lines in tissue culture media; microscopy of cultured cells; propagation of herpesviruses in tissue culture; and recognition of cytopathic effects.

- To attain a basic understanding of techniques important to immune function and phenotype assays, including flow cytometry, cell proliferation assays, antigen stimulation, interferon-gamma (IFN- $\gamma$ ) production and enzyme-linked immunosorbent spot (ELISPOT) assays, and immunohistochemistry.

- To achieve a detailed understanding of molecular methods used to diagnose viral infections (CMV, EBV, $\mathrm{BKV}$, respiratory viruses) in SOT/HSCT patients, including the use of positive/negative controls, assay 
variability, appropriate specimens for testing, and clinical interpretation of results.

\section{Research training: translational, basic, and clinical research (individualized)}

The research training should be focused on the individual goals of the fellow and may incorporate additional time beyond the core year of training to complete more advanced research training. Depending on the goals, research may be basic, translational, or clinical in nature.

- Basic science training may involve mastering laboratory techniques that are fundamental to transplant ID including sterile technique, centrifuging, pipetting, running gels, making media, and filtering. Additional virology techniques could include maintenance of cell lines in tissue culture media and microscopy of cultured cells, propagation of herpesviruses in tissue culture, and recognition of cytopathic effects. Upon mastering these skills, they would be applied to an appropriate research project developed in conjunction with and supervised by the attending physicians.

- Translational research may include mastering the techniques above as well as techniques to assess immune function and phenotype assays, such as flow cytometry, cell proliferation assays, antigen stimulation, IFN $\gamma$ production and ELISPOT assays, and immunohistochemistry. Upon mastering these skills, they would be applied to an appropriate research project developed in conjunction with and supervised by the attending physicians.

- Clinical research may include designing and completing a high-quality retrospective chart or database review of a crucial and under-reported topic in transplant ID or a high-quality systematic review of a crucial and under-reported topic in transplant ID.

\section{Objectives}

- All fellows should have training in the design, implementation, data collection, analysis, and reporting of a basic-translational study with relevance to transplant infections.
- An abstract should be submitted for presentation at a national/international transplant ID-related meeting. A summary manuscript should be drafted and, ideally, submitted for publication. Attendance at a transplant ID-related meeting is strongly encouraged.

\section{Evaluation}

At the end of each rotation (monthly), oral or written evaluation by the transplant ID staff physician or laboratory/research supervisor should include an assessment of all standard training competencies (knowledge, practicebased learning, professionalism, ethics, and systems development) as related to transplant ID.

$\diamond$ Optimally, multi-source feedback on inter-professional, collaborator, and communication skills should be sought from transplant ID staff as well as all members of the multi-disciplinary transplant team.

- Knowledge and knowledge application should be assessed at or before the midpoint of training, using oral or written examinations, including a series of shortanswer questions available on the AST website.

- Feedback should be sought from the fellow on the transplant ID training program, transplant ID staff, and advisors.

\section{Summary}

This curriculum serves to provide comprehensive guidelines for training fellows in transplant ID. These guidelines will no doubt need to be modified for the unique environment of each transplant center. Moreover, as the field changes and new paradigms in transplantation emerge, updates will be required as well.

\section{Acknowledgements:}

We would like to thank Drs Robin Avery and Jay Fishman for generously providing the original document that served as the basis for this curriculum and for their ongoing guidance to the AST ID Educational Initiatives Working Group. 